\title{
Duality of massive gauge invariant theories in arbitrary space-time dimension*
}

\author{
A.Smailagic ${ }^{\dagger}$ \\ Department of Physics, Faculty of Electrical Engineering \\ University of Osijek, Croatia \\ E. Spallucci ${ }^{\ddagger}$ \\ Dipartimento di Fisica Teorica \\ Università di Trieste, \\ INFN, Sezione di Trieste
}

\begin{abstract}
We show that dualization of Stueckelberg-like massive gauge theories and $B \wedge F$ models, follows form a general $p$-dualization of interacting theories in $d$ spacetime dimensions. This is achieved by a particular choice of the external current.
\end{abstract}

\footnotetext{
*Accepted for publication in Phys.Rev.D

${ }_{\dagger}$ E-mail address: anais@etfos.hr

$\ddagger$ E-mail address:spallucci@trieste.infn.it
} 
Recently, various models of $B \wedge F$ theories in $3+1$ dimensions, either in relation to the Bosonization of fermionic models [四], or with respect to their dual Stueckelberg-like gauge models [2], have been considered. In this note we would like to show how to dualize $B \wedge F$ models in a general way. Also we show that such dualization is a special case of a general formalism of $p$-dualization of interacting field theories. Here we give a brief review of the general $p$-dualization procedure proposed in [3]. As usual, one defines a parent Lagrangian from which original and dual theories can be obtained. Parent Lagrangians are not uniquely defined and there exist various methods for their constructions [4]. One of commonly used method is based on a "shift symmetry" which has been also adopted in [2]. Instead we choose in our approach, what we believe to be, a simpler and more transparent formulation of parent Lagrangian. It will describe dualization of any interacting, theory of $p$-forms in arbitrary spacetime dimension [3]. Let us start with a theory of a potential $p$-form $V(x)$ in arbitrary dimensions $d$ interacting with an a priori external current $K(x)$. The dual field $H$ is a $d-p-1$ form with the rank $p$ as $p \leq d-1$. Parent Lagrangian is constructed as

$$
\begin{aligned}
L_{P}= & -\frac{1}{2(d-p-1) !} H_{\mu_{1} \ldots \mu_{d-p-1}} H^{\mu_{1} \ldots \mu_{d-p-1}}+\frac{1}{(d-p-1) !} H^{\mu_{1} \ldots \mu_{d-p-1}} F^{*}{ }_{\mu_{1} \ldots \mu_{d-p-1}}(V) \\
& +\frac{1}{(d-p-1) !} K^{\mu_{1} \ldots \mu_{d-p-1}} H_{\mu_{1} \ldots \mu_{d-p-1}}
\end{aligned}
$$

with the notation $F^{* \mu_{1} \ldots \mu_{d-p-1}}(V)=\frac{1}{(p+1) !} \epsilon^{\mu_{1} \ldots \mu_{d-p-1} \mu_{d-p} \ldots \mu_{d}} F_{\mu_{d-p} \ldots \mu_{d}}(V)$ and $F_{\mu_{d-p} \ldots \mu_{d}}(V) \equiv \partial_{\left[\mu_{d-p}\right.} V_{\left.\mu_{d-p+1} \mu_{d}\right]}$. Since, in general, dual field $H$ can be also a gauge potential the external current $K$ is not conserved, i.e. $\partial K \neq 0$.

Dualization proceeds in the following way:

i) varying the parent Lagrangian with respect to the field $H$ leads to an "equation of motion"

$$
\delta_{H} L_{P}=0 \longrightarrow H^{\mu_{1} \ldots \mu_{d-p-1}}=F^{* \mu_{1} \ldots \mu_{d-p-1}}(V)+K^{\mu_{1} \ldots \mu_{d-p-1}}
$$

The above equation, inserted back into (1), gives the interacting theory for the $V$ field described by the Lagrangian

$$
\begin{aligned}
L_{V, K} & =\frac{1}{2(p+1) !}\left[F^{*}{ }_{\mu_{1} \ldots \mu_{p+1}}(V)+K_{\mu_{1} \ldots \mu_{p+1}}\right]^{2} \\
& =-\frac{1}{2(p+1) !}\left[F_{\mu_{1} \ldots \mu_{p+1}}(V)-(-1)^{(p+1)(d-p-1)} K^{*}{ }_{\mu_{1} \ldots \mu_{p+1}}\right]^{2}
\end{aligned}
$$


where, the $K^{*}$ is the Hodge dual of the current $K$, and the factor $(-1)^{(p+1)(d-p-1)}$ comes from epsilon contractions. One sees from (3) that there is a current contact term in the theory for $V$. Such terms are unavoidable products of dualization procedure, and it turns out that they are necessary to make dualization consistent [5], [6]. They will turn out to be essential for what we are going to prove.

ii) The dual theory, on the other hand, is obtained varying parent Lagrangian with respect to the field $V$ which gives "equation of motion" as

$$
\delta_{V} L=0 \quad: \quad \partial^{\mu_{1}} H^{*}{ }_{\mu_{1} \ldots \mu_{p+1}}=0
$$

which has solution

$$
H_{\mu_{1} \ldots \mu_{d-p-1}}=\partial_{\left[\mu_{1}\right.} B_{\left.\mu_{2} \ldots \mu_{d-p-1}\right]} \equiv H_{\mu_{1} \ldots \mu_{d-p-1}}(B)
$$

Therefore, the dual field $H$ is defined through (四), and it turns out to be a field strength of the dual potential $B$. (5) inserted back in (14) gives the dual theory for the field $B$, coupled to an external current $K$

$$
\begin{aligned}
L_{B, K}= & -\frac{1}{2(d-p-1) !} H_{\mu_{1} \ldots \mu_{d-p-1}}(B) H^{\mu_{1} \ldots \mu_{d-p-1}}(B)+ \\
& +\frac{1}{(d-p-1) !} K^{\mu_{1} \ldots \mu_{d-p-1}} H_{\mu_{1} \ldots \mu_{d-p-1}}(B)
\end{aligned}
$$

The end result of our dualization procedure is that to any interacting theory of the $p$ form $V$, described by the Lagrangian (3), corresponds a dual interacting theory in terms of the dual potential $B$, described by the Lagrangian (6). One can verify that this procedure reproduces all known dual theories as described for example in 四 (see final discussion). At this point one may wonder how can this procedure produce dualization of $B \wedge F$ theories? Before we address the above question we rewrite, by partial integration, the interaction term of the dual theory as

$$
\begin{aligned}
L_{B, \text { int. }} & \equiv \frac{1}{(d-p-1) !} K^{\mu_{1} \ldots \mu_{d-p-1}} H_{\mu_{1} \ldots \mu_{d-p-1}}(B) \\
& =-\frac{1}{(d-p-2) !}\left(\partial_{\mu_{1}} K^{\mu_{1} \mu_{2} \ldots \mu_{d-p-1}}\right) B_{\mu_{2} \ldots \mu_{d-p-1}}
\end{aligned}
$$


Also notice that the described dualization procedure will not be affected if one adds to the parent Lagrangian (11) any function of the external current $K$. Therefore we choose to modify (11), for the reason to become clear soon, as

$$
L_{P} \longrightarrow L_{P}+\frac{1}{2 m^{2}(d-p-2) !}\left(\partial_{\mu_{1}} K^{\mu_{1} \mu_{2} \ldots \mu_{d-p-1}}\right)^{2}
$$

and a mass parameter $m$ has to be introduced for dimensional reasons.

What we claim is that the general interaction term (7) is equivalent to the $B \wedge F$ terms in special cases. To see this, let us rewrite the external current $K$ as the Hodge dual of a new gauge field $A$. By "new gauge field" we mean independent of the gauge fields $V$ and $H$, which are subject to the dualization procedure.

$$
K^{\mu_{1} \ldots \mu_{d-p-1}}=\frac{m}{(p+1) !} \epsilon^{\mu_{1} \ldots \mu_{d-p-1} \mu_{d-p} \ldots \mu_{d}} A_{\mu_{d-p} \ldots \mu_{d}}
$$

Inserting (9) into (7) we find that the interaction term $H(B) K$ translates into

$$
L_{B, \text { int. }} \equiv \frac{m}{d !} \epsilon^{\mu_{1} \ldots \mu_{d}} B_{\mu_{1} \ldots \mu_{d-p-2}} \partial_{\left[\mu_{d-p-1}\right.} A_{\left.\mu_{d-p} \ldots \mu_{d}\right]} \equiv m B^{\mu_{1} \ldots \mu_{d-p-2}} F^{*}{ }_{\mu_{1} \ldots \mu_{d-p-2}}(A)
$$

which is a generalized $B \wedge F$ term. $\mathcal{Q E D}$. Then, the Lagrangian (3) of the original theory for $V$ becomes

$$
L_{V, A}=-\frac{1}{2(p+1) !}\left(\partial_{\left[\mu_{1}\right.} V_{\left.\mu_{2} \ldots \mu_{p+1}\right]}+m A_{\mu_{1} \ldots \mu_{p+1}}\right)^{2}-\frac{1}{2(p+2) !} F_{\mu_{1} \ldots \mu_{p+2}}^{2}(A)
$$

(11) makes clear the choice of the function $f(K)$, previously introduced in the parent Lagrangian. It gives a kinetic term of the new gauge field $A$. Therefore, starting from a general interacting theory, we obtain gauge invariant, Stueckelberg-like Lagrangian, of a massive $p+1$-form potential $A$, where the starting $p$-form $V$ field acts as a Stueckelberg conpensator.

The dual $B \wedge F$ theory of such Stueckelberg-like model follows directly from (6) as

$$
\begin{aligned}
L_{B F}= & -\frac{1}{2(d-p-1) !} H_{\mu_{1} \ldots \mu_{d-p-1}}^{2}(B)+\frac{1}{d !} \epsilon^{\mu_{1 \ldots} \ldots \mu_{d}} B_{\mu_{1} \ldots \mu_{d-p-2}} \partial_{\left[\mu_{d-p-1}\right.} A_{\left.\mu_{d-p} \ldots \mu_{d}\right]}+ \\
& -\frac{1}{2(p+2) !} F_{\mu_{1} \ldots \mu_{p+2}}^{2}(A)
\end{aligned}
$$


This is the general result we were set to prove in this note.

To make the final result more transparent let us look at specific case of $(3+1)$ dimensions. We find the following models:

1. choice of rank $p=0$ implies identification of various fields as $V \rightarrow \Phi, B \rightarrow B_{\mu \nu}$, $A \rightarrow A_{\mu}$. From the above formulae one finds the Stueckelberg gauge theory of the massive vector field as

$$
L_{\Phi}=-\frac{1}{2}\left(\partial_{\mu} \Phi+m A_{\mu}\right)^{2}-\frac{1}{4} F_{\mu \nu}^{2}(A)
$$

and its dual $B \wedge F$ theory, in terms of the Kalb-Ramond tensor field, which is given by the Lagrangian

$$
L_{B}=-\frac{1}{2 \cdot 3 !} H_{\mu \nu \rho}^{2}(B)+\frac{m}{4 !} \epsilon^{\mu \nu \rho \sigma} B_{\mu \nu} \partial_{[\rho} A_{\sigma]}-\frac{1}{4} F_{\mu \nu}^{2}(A)
$$

2. choice of rank $p=1$ implies identification of various fields as $V \rightarrow \Phi_{\mu}, B \rightarrow B_{\mu}$, $A \rightarrow$ $A_{\mu \nu}$. Stueckelberg-like formulation of the massive, gauge invariant, Kalb-Ramond model is given by

$$
L_{A}=-\frac{1}{4}\left(\partial_{[\mu} \Phi_{\nu]}+m A_{\mu \nu}\right)^{2}-\frac{1}{2 \cdot 3 !} F_{\mu \nu \rho}^{2}(A)
$$

while the dual $B \wedge F$ theory is described by the Lagrangian

$$
L_{B}=-\frac{1}{4} H_{\mu \nu}^{2}(B)+\frac{m}{4 !} \epsilon^{\mu \nu \rho \omega} B_{\mu} \partial_{[\nu} A_{\rho \sigma]}-\frac{1}{2 \cdot 3 !} F_{\mu \nu \rho}^{2}(A)
$$

3. Last possible choice is $p=2$, which implies $V \rightarrow \Phi_{\mu \nu}, B \rightarrow \phi, A \rightarrow A_{\mu \nu \rho}$ leading to Stueckelberg Lagrangian

$$
L_{A}=-\frac{1}{2 \cdot 3 !}\left(\partial_{[\mu} \Phi_{\nu \rho]}+m A_{\mu \nu \rho}\right)^{2}-\frac{1}{2 \cdot 4 !} F_{\mu \nu \rho \sigma}^{2}(A)
$$

while the dual $B \wedge F$ theory is described by the Lagrangian 


$$
L_{B}=-\frac{1}{2}\left(\partial_{\mu} \phi\right)^{2}+\frac{m}{4 !} \epsilon^{\mu \nu \rho \sigma} \phi \partial_{[\mu} A_{\nu \rho \sigma]}-\frac{1}{2 \cdot 4 !} F^{2}{ }_{\mu \nu \rho \sigma}(A)
$$

and so on ad nauseam, for other spacetime dimension.

The first and the last models in the above examples are exactly those described in [2] while we showed that the general formalism gives also a third model based on vectorto-vector gauge field dualization. As a conclusion, we have shown how Stueckelberg-like massive gauge theories of an external gauge potential $A$, introduced as the Hodge dual of the external current $K$, and their dual $B \wedge F$ models, represent special cases of a general dualization procedure of interacting theories. Realization of Stueckelberg-like model is due to the fact that dualization procedure combines the field strength of the potential $V$ and the external current $K$ in the form ([3), thanks to the existence of the contact term $K^{2}$. Our general procedure thus enables to consider dual theories at will by appropriate choice of the external current. Note that the choice $A=0$ in the Stueckelberg-like four dimensional examples reproduces all known four dimensional dual free theories, i.e. scalar to KalbRamond (either way), and vector to vector. This proves the power and generality of the proposed method. 


\section{REFERENCES}

[1] R.Banerjee, C.Marino Phys.Rev.D 56, 3763 (1997)

[2] E.Harikumar, M. Sivakumar Phys.Rev.D 57, 3794 (1998)

[3] A.Smailagic, E.Spallucci " Dualization of Interacting Theories Including $p=d-1$ Limiting Cases" preprint, (1999)

[4] S.E.Hjelmeland, U.Lindstrom, "Duality for the Non-Specialist" hep-th/9705122

[5] S.Deser, R.Jackiw, Phys. Lett. 139B, n.5,6, 371 (1984)

[6] Burgess C.P. Burgess, M.T.Grisaru,M.Kamela, M.E. Knutt-Wehlau, P. Page, F.Quevedo and M.Zebarjad Nucl.Phys.B542, 195, (1999) 